\title{
Isotope Dependence of the Chemical Erosion of Graphite by Hydrogen/Deuterium Implantation
}

\author{
Egon VIETZKE* \\ Institut für Plasmaphysik, Forschungszentrum Jülich GmbH, Euratom Association, \\ Trilateral Euregio Cluster, D-52425 Jülich, Germany \\ (Received October 4, 2001 and accepted in revised form December 19, 2001)
}

\begin{abstract}
Since graphite material is still a candidate for parts of planned fusion devices as ITER, the chemical erosion by hydrogen isotopes is a major concern.

At energies of the impinging hydrogen below $100 \mathrm{eV}$, a strong isotope effect of the chemical erosion of carbon at low temperature is observed by hydrogen and deuterium ion impact. This effect is diminished at elevated temperatures around $800 \mathrm{~K}$. For energies of the impinging hydrogen ion around $200 \mathrm{eV}$, only a minor isotope effect is observed in the maximum erosion yield at temperatures around $750 \mathrm{~K}$. At low temperatures, the chemical erosion yields for these ion energies are much smaller than those for ions below $100 \mathrm{eV}$. Hydrogen/deuterium ions with energies of $1 \mathrm{keV}$ and above show no isotope effect at all.

Also in fusion devices as JET, TEXTOR and JT-60 an isotope effect in the chemical erosion of carbon materials is observed. The erosion yield in deuterium plasmas $\left(T_{e}=10-50 \mathrm{eV}\right)$ is $1.3-1.5$ of that in a hydrogen plasma.

The multi-step process of chemical erosion of carbon materials by hydrogen isotopes is reasonably understood. The isotope effect is mainly caused by the radiation damage of the incident ions and by the ion-induced release process.
\end{abstract}

KEYWORDS: isotope effects, chemical erosion, hydrogen, deuterium, graphite, carbon materials, implantation, eV range 01-100, temperature dependence

\section{Introduction}

The chemical erosion of graphite or any carbon-based material by hydrogen isotopes is an important problem in the field of plasma-surface interaction of nuclear fusion. The most promising fusion reaction is that of deuterium and tritium leading to a $14 \mathrm{MeV}$ neutron and a $3.6 \mathrm{MeV}$ alpha particle and after neutralization to a helium atom. The most advanced fusion device is the tokamak. A major concern of the tokamak is the high particle load concentrated on limiters or divertor plates. Such high particle fluxes are favorable for helium pumping, but problematic for heat removal and for the erosion by the particles. Carbon material is used in the present day tokamaks for such high heat load components and is still a candidate for the divertor plates of the planned fusion devices as ITER due to its good thermomechanical properties. ${ }^{1)}$ A major concern is the chemical erosion and as a consequence the tritium retention via codeposition of the formed hydrocarbons. For an understanding and modeling of the impurity content and transport in the plasma boundary of fusion devices the impurity sources have to be known, especially the formation yields and their dependence on the impinging hydrogen isotopes as the main plasma constituents. For the modeling of the behavior in fusion reactors, most of the data have to be extrapolated from hydrogen/deuterium behavior to the dependence on tritium. In this paper, an overview will be given on the isotopic effects in the chemical erosion of graphite by hydrogen and deuterium ion impact.

Chemical erosion of carbon materials by hydrogen is a complicated multi-step process and depends on the energy and flux density of the impinging hydrogen, surface tempera-

*Corresponding author, Tel. +49-2461613113, Fax. +492461612660,E-mail: E.Vietzke@fz-juelich.de ture and material properties. ${ }^{2-4)}$ Such complex behavior cannot be studied in a fusion devise, it has to be investigated in well defined ion or atom beam experiments. ${ }^{5}$ The total erosion is hereby determinated by the weight loss of the target or by eroding a certain thickness with respect to the incident ion fluence. The reaction product spectrum is mostly determined by mass spectrometry either in the residual gas or directly in a line-of-sight, differentially pumped quadrupole mass spectrometer. ${ }^{6}$ ) In certain cases also optical spectroscopy has been applied especially emission spectroscopy in front of limiters or of divertor plates. ${ }^{7)}$ For a determination of the hydrocarbon formation, $\mathrm{CH}(\mathrm{CD})$ bands around $430 \mathrm{~nm}$ are used in relation to $\mathrm{H}_{\alpha} \mathrm{H}_{\gamma}\left(\mathrm{D}_{\alpha}\right.$ or $\left.\left.\mathrm{D}_{\gamma}\right) .{ }^{8}\right)$ However, the $\mathrm{CD}$ bands are overlaid by $\mathrm{D}_{2}$ bands depending on plasma conditions. An overestimation of the CD light by $20 \%$ is possible. ${ }^{9)}$

\section{Results on Isotope Effects in the Chemical Erosion}

\section{Results from Ion Beam Experiments}

In Fig. 1 the total erosion yield of graphite at room temperature by $\mathrm{H}^{+} / \mathrm{D}^{+}$ion beam impact is shown as a function of incident ion energy by weight loss measurements. ${ }^{10)}$ The chemical erosion yield is the difference between physical sputtering (solid lines) and the measured total erosion. Below $100 \mathrm{eV}$, a clear isotope effect of the chemical erosion of carbon is observed by hydrogen and deuterium ion implantation. Furthermore, the chemical erosion at room temperature is negligible for incident energies above $300 \mathrm{eV}$. Within the scatter of the data these results are confirmed in later experiments also by weight loss. ${ }^{11)}$

The temperature dependence of the chemical erosion for $200 \mathrm{eV} \mathrm{H}^{+}\left(\mathrm{D}^{+}\right)$and for low energetic $\mathrm{H}^{+}\left(\mathrm{D}^{+}\right)(10$ and $15 \mathrm{eV})$ 


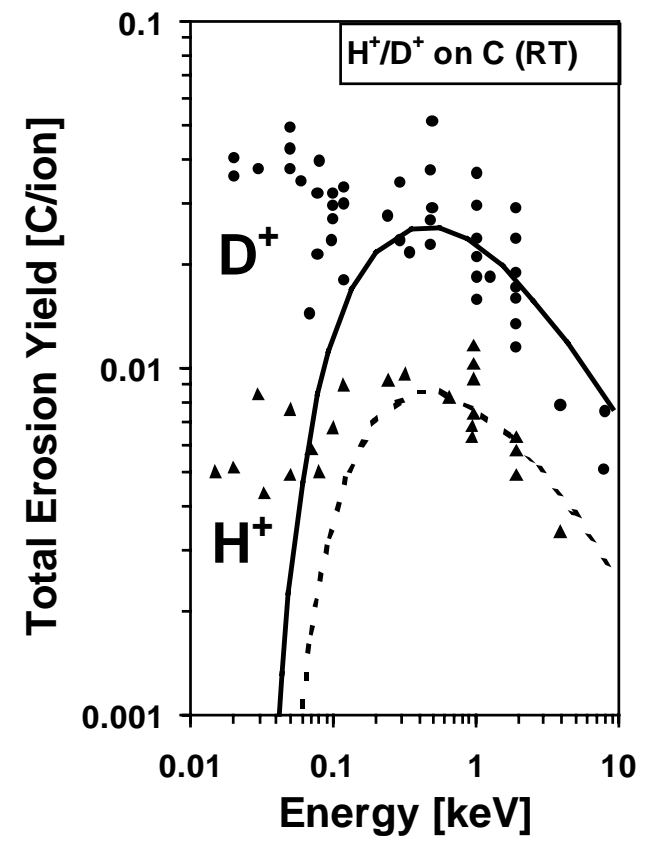

Fig. 1 Energy dependence of the total erosion yield (chemical erosion and physical sputtering) of graphite at room temperature by $\mathrm{H}^{+}\left(\mathrm{D}^{+}\right)$

The lines are calculated physical sputtering yields. ${ }^{10)}$

shown in Fig. 2 is obtained by two different methods: by analyzing the reaction product spectrum in the residual gas in Fig. 2(a) ${ }^{12)}$ and by weight loss measurements in Fig. 2(b). ${ }^{11)}$ The isotopic effect in both sets of curves follows the same trend. For low energetic $\mathrm{H}^{+}\left(\mathrm{D}^{+}\right)$, there is a nearly constant isotope effect in the yields from $300 \mathrm{~K}$ up to the maximum. At temperatures above the maximum yield this effect is diminished. For $200 \mathrm{eV} \mathrm{H}^{+}\left(\mathrm{D}^{+}\right)$, the yield at $300 \mathrm{~K}$ is nearly equal for both isotopes; but in the maximum around $750 \mathrm{~K}$ the yield for $\mathrm{D}^{+}$is $50 \%$ larger than for $\mathrm{H}^{+}$. Not shown here, for energies at $1 \mathrm{keV}$ and above, also the isotope effect in the maximum vanishes. ${ }^{11)}$ However, the absolute yields by the weight loss method in Fig. 2(b) are always around a factor of two higher than the results obtained by mass spectrometry in the residual gas. Furthermore, the isotope effect in the chemical erosion for low energetic $\mathrm{H}^{+}\left(\mathrm{D}^{+}\right)$at $300 \mathrm{~K}$ observed by mass spectrometry is smaller $\left(Y_{\mathrm{D}^{+}} \approx 2 \times Y_{\mathrm{H}^{+}}\right)$than that by weight loss $\left(Y_{\mathrm{D}^{+}} \approx 6 \times Y_{\mathrm{H}^{+}}\right)$. In Ref. 11) is shown that a fraction of the chemically eroded particles (e.g. radicals) stick to the vessel surface and will not reach the residual gas. This explains, that the chemical erosion yield obtained by mass spectrometry in the residual gas is lower than that by weightloss.

\section{Results in Fusion Devices}

Using CD emission spectroscopy at a externally heated test limiters, the $\mathrm{CD}_{4}$ formation was measured as function of the target temperature. ${ }^{13)}$ The yield is between 3-5\% and the temperature dependence is more flat until $1,000 \mathrm{~K}$ compared to ion beam results. But in principle, the temperature dependence is comparable to ion beam results.

Very recently, an isotope effect in the total chemical ero-
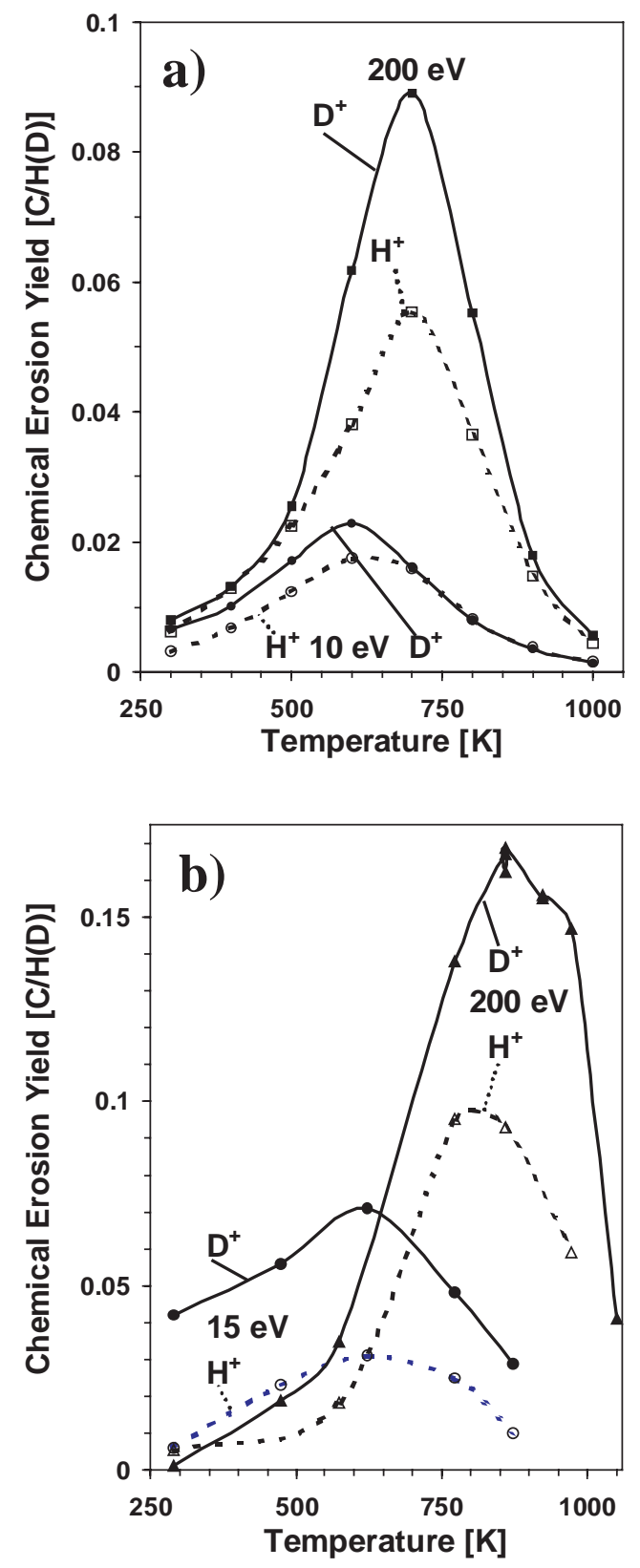

Fig. 2 Total chemical erosion yield by $\mathrm{H}^{+}\left(\mathrm{D}^{+}\right)$impact on graphite as a function of specimen temperature for 200,15 and $10 \mathrm{eV}$ ion energy obtained by two different methods: by analyzing the reaction product spectrum in the residual gas in Fig. 2(a) ${ }^{12)}$ and by weight loss measurements in Fig. 2(b) ${ }^{11)}$

The flux densities were between $1-8 \times 10^{19} / \mathrm{m}^{2} \cdot \mathrm{s}$. The lines through the data serve to aid the reader.

sion or the divertor plate by hydrogen and deuterium plasmas is observed in the fusion experiment JET (see Fig. 3) ${ }^{14}$ using again emission spectroscopy and the newest results on the CD-band structure. ${ }^{9)}$ Even after correcting the possible overestimation of the $\mathrm{CD}$ bands an isotope effect remains, the yield ratio is $Y^{\mathrm{D}} / Y^{\mathrm{H}}=1.3-1.7$. An similar result is seen in TEXTOR by spectroscopy of the emitted light from a test limiter $^{15)}$ which is easier accessible. Also Kubo et al. report an observed isotope effect in the chemical erosion in the divertor of JT-60. ${ }^{16)}$ 


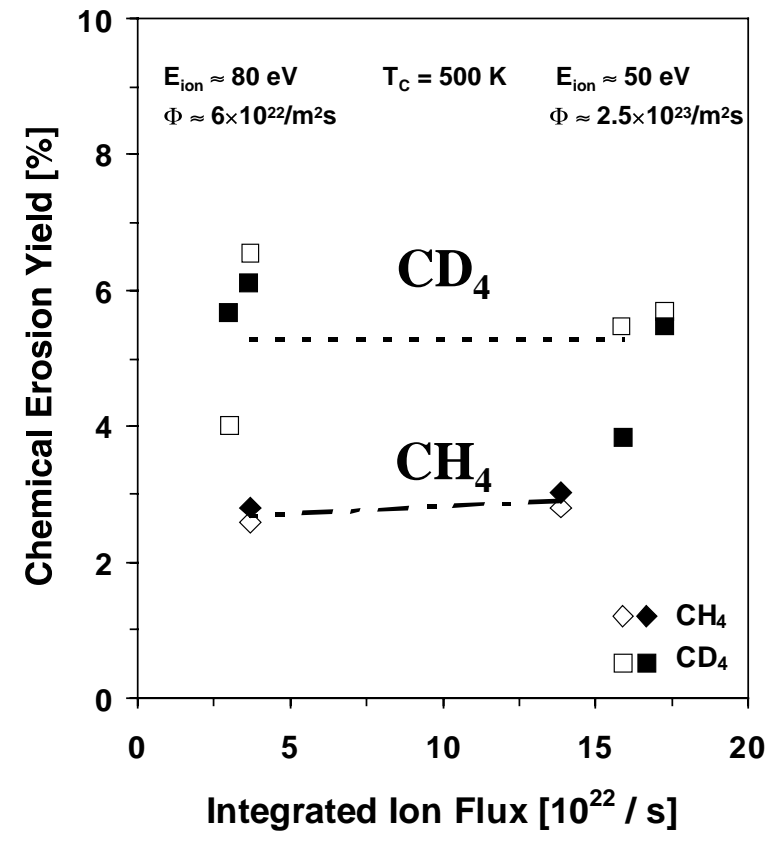

Fig. 3 Hydrocarbon erosion yields as a function of the integrated ion fluxes from $\mathrm{H}$ and $\mathrm{D}$ plasmas to the outer divertor

The incident flux is calculated from Langmuir probes (solid points) and from $\mathrm{D}_{\alpha}$ (open points). ${ }^{14)}$

\section{The Reaction Mechanism}

The chemical erosion of carbon by impact of hydrogen or its isotopes (in the following I will only use the expression hydrogen meaning mostly hydrogen and its isotopes) is a multi-step process. ${ }^{2,4)}$ Obviously, two branches are responsible for the hydrocarbon formation: the so called surface reaction with the yield $Y_{\text {surf }}$ at low temperature and low energetic hydrogen ions and the thermal reaction with $Y_{\text {therm }}$ around the maximum. The 1st step for both branches is a modification of the near surface layer up to the implantation depth. The radiation damage provides open bonds and the thermalized hydrogen ions react on open bonds to form $\mathrm{CD}_{3}-\mathrm{C}$ complexes.

The mechanism of the thermal reaction branch is relatively early proposed ${ }^{17)}$ and later detailed elucidated by Küppers and his group, ${ }^{18,19)}$ see Fig. 4. The reaction occurs at inner surfaces at the end of the implantation depth where the thermalized hydrogen atom undergoes an abstraction reaction, forming $\mathrm{H}_{2}$ and an intermediate radical center which can above $400 \mathrm{~K}$ release $\mathrm{CH}_{3}$. The maximum of this reaction is reached around $700 \mathrm{~K}$ and-important for our discussionno dependence on the hydrogen isotope is observed for this reaction step. ${ }^{20)}$ The final step occurs on the penetration to the surface. The $\mathrm{CH}_{3}$ adsorbed at inner surfaces is released as $\mathrm{CH}_{4}$ from the adsorbed state ion-induced as well as thermally driven. ${ }^{20,22)}$ The observed isotope effect is explainable by the radiation damage depending on the ion mass or by the part which is released by ion impact.

The mechanism of the surface reaction is not understood in detail, but can be described quite simply. After the modification of the surface in a 1 st step, $\mathrm{CD}_{4}$ is mainly relased by ion impact from near surface $\mathrm{CD}_{3}-\mathrm{C}$ complexes. This ion-induced effect is not a physical sputtering process which
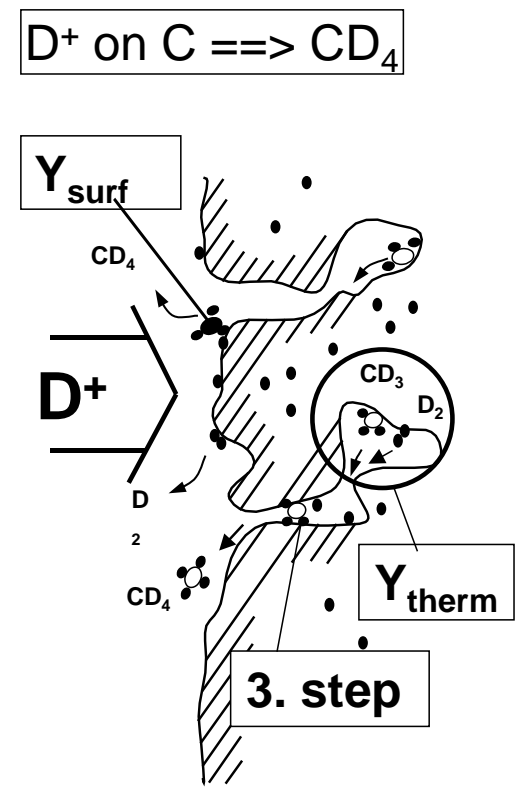

Fig. 4 Schematic model of the hydrocarbon formation in carbon by deuterium ion impact within two branches: the thermal reaction around the maximum of $700 \mathrm{~K}$ and the surface reaction for low energetic D-ions and at low temperatures

The first step for both branches: Radiation damage provides open bonds on which thermalized $\mathrm{D}$-ions form $\mathrm{CD}_{3}-\mathrm{C}$ complexes. In the thermal reaction, the 2nd and main step is the release of $\mathrm{CD}_{3}$ after $\mathrm{D}_{2}$ abstraction above $400 \mathrm{~K}$ at the implantation depth. The final step in the thermal reaction is the ion-induced and thermal release of $\mathrm{CD}_{4}$ from $\mathrm{CD}_{3}$-adsorbed complexes during the penetration to the surface. In the surface reaction, the second and final step is the ion-induced release of $\mathrm{CD}_{4}$ from near surface $\mathrm{CD}_{3}-\mathrm{C}$ complexes.4,21,22)

would result in a different energy distribution as observed in Ref. 22) and would not be reduced with increasing hydrogen ion energy. Most probably it is a direct hydrogen ion reaction with near surface $\mathrm{CD}_{3}-\mathrm{C}$ complexes. The isotope effect of the surface reaction can be explained by the radiation damage depending on the ion mass and by the ion-induced release from near surface complexes.

\section{Conclusion}

In modeling the chemical erosion at the divertor plate of a fusion reactor many aspects have to be taken into account. One important problem is the extrapolation of measured erosion yields to high particle flux densities expected for a fusion reactor divertor especially whether or not a flux density dependence exists with reduces yields at high values. ${ }^{23}$ ) The other unsolved problem is the deposition of hydrocarbon molecules on the divertor plate and their neighborhood leading to an unacceptable tritium inventory.

From the above reviewed results we have to conclude: a clear isotope effect exists in the chemical erosion of carbon materials by hydrogen and deuterium and has to be taken into account in modeling the chemical erosion of graphite in a deuterium-tritium plasma. 


\section{References}

1) G. Federici, R. A. Andrea, P. Andrew, et al., J. Nucl. Mater., 266-269, 14 (1999).

2) E. Vietzke, A. A. Haasz, "Chemical erosion," Physical Processes of the Interaction of Fusion Plasmas with Solids, (W. O. Hofer, J. Roth, ed.) Academic Press, Amsterdam, p. 135 (1996).

3) J. W. Davis, A. A. Haasz, J. Nucl. Mater, 241-243, 37 (1997).

4) J. Roth, J. Nucl. Mater., 266-269, 51 (1999).

5) J. Roth, E. Vietzke, A. A. Haasz, Suppl. Nucl. Fusion, 1, 63 (1991).

6) E. Vietzke, K. Flaskamp, V. Philipps, J. Nucl. Meter., 111-112, 793 (1982)

7) A. Pospieszczyk, Atomic and Plasma-Material Interaction Processes in Controlled Thermonuclear Fusion, (R. K. Janev, H. W. Drawin, ed.) Elsvier, Amsterdam, (1993).

8) A. Pospieszczyk, G. Sergienko, D. Rusbüldt, Contr. Plasma Phys., 40, 162 (2000).

9) S. Brezinsek, Ph. Mertens, A. Pospieszczyk, et al., Proc. 1st Workshop on Hydrogen in the Plasma Edge (1st WHYPE),
Jülich 2000; Submitted to Contr. Plasma Phys.

10) J. Roth, J. Bohdansky, Nucl. Instrum. Methods, B23, 549 (1987).

11) M. Balden, J. Roth, J. Nucl. Mater., 280, 39 (2000).

12) B. V. Mech, A. A. Haasz, J. W. Davis, J. Nucl. Mater, 225, 153 (1998).

13) A. Pospieszczyk, V. Philipps, E. Casarotto, et al., J. Nucl. Mater., 241-243, 833 (1997).

14) M. F. Stamp, et al., J. Nucl. Mater., 290-293, 321 (2001).

15) A. Pospieszczyk, et al., To be published.

16) H. Kubo, et al., ITER Meeting at JAERI, July 2001.

17) E. Vietzke, K. Flaskamp, V. Philipps, J. Nucl. Mater., 128-129, 545 (1984).

18) A. Horn, A. Schenk, J. Biener, et al., Chem. Phys. Lett., 231, 3125 (1993).

19) J. Küppers, Surf. Sci. Rep., 22, 249 (1995).

20) J. Biener, Ph. D. Thesis, University of Bayreuth, (1964).

21) E. Vietzke, V. Philipps, Fusion Technol., 15, 108 (1989).

22) E. Vietzke, J. Nucl. Mater., 290-293, 158 (2001).

23) J. Roth, Physica Scripta, T91, 65 (2001). 